\title{
AUTOCUIDADOS Y EDUCACIÓN TERAPÉUTICA EN DIABETES. UNA REVISIÓN BIBLIOGRÁFICA.
}

\section{SELF CARE AND THERAPEUTIC EDUCATION IN} DIABETES. A LITERATURE REVIEW.

Víctor Manuel González Chordáa,e

Pablo Salas Medina ${ }^{b, e}$

Desirée Mena Tudelac,e

Alexandra Gimeno Cardells ${ }^{c}$
a. Instituto de Traumatología de Unión de Mutuas.
b. Sociedad de Anestesistas de Castellón.
c. IIS La Fe, Valencia.
e. Programa de doctorado en Ciencias de la Salud de la Universitat Jaume I.

E-mail: vmgc81@gmail.com 


\section{RESUMEN}

\section{Título:}

Autocuidados y educación terapéutica en diabetes. Una revisión bibliográfica.

\section{Objetivos:}

La Educación Terapéutica en Diabetes (ETD) influye en su la variabilidad pronóstica. El rigor del profesional de la salud debería ser el mismo tanto prescribiendo fármacos como dando normas de conducta. El objetivo del presente estudio fue revisar la literatura reciente relacionada con la diabetes, la educación terapéutica y los autocuidados.

\section{Metodología:}

Se realizó una búsqueda bibliográfica en las bases de datos PudMed, Cuiden, Ibecs, Lilacs, Scielo y Enfispo en febrero de 2012. El límite temporal fue de 5 años. Se buscaron las palabras clave en título, abstract y como descriptores.

\section{Resultados:}

Se identificaron un total de 194 artículos. El 29\% se incluyeron en el estudio $(n=54)$. El $64.4 \%$ de los trabajos fueron localizados en PudMed $(n=124)$; no se encontró ningún resultado en la base de datos CUIDEN. El principal motivo de exclusión fue que los artículos no estaban relacionados directamente con el tema de la revisión en el $32.8 \%$ de los casos $(n=65)$. Los trabajos con carácter cualitativo fueron los más abundantes $(n=12)$.

\section{Conclusiones:}

La implantación de intervenciones educativas innovadoras centradas en los autocuidados parece tener beneficios sobre los pacientes diabéticos, mejorando el manejo de la enfermedad o las cifras de glucosa y hemoglobina glicosilada, facilitando el empowerment de los pacientes o diminuyendo el control paterno en diabéticos tipo I. Es necesaria más investigación experimental que aclare qué tipo de intervenciones educativas presentan mayores ventajas y beneficios para las personas diabéticas en diferentes contextos.

\section{Palabras clave:}

diabetes mellitus, diabetes tipo I, diabetes tipo II, educación, autocuidados. 


\section{ABSTRACT}

\section{Title:}

Self-care and therapeutic education in diabetes. A literature review.

\section{Aims:}

The therapeutic education in Diabetes (NTDS) influences in its variability prognostic, highlighting the fact that the rigour of the health professional should be the same both prescribing drugs as giving rules of conduct. The objective of the present study was to review the recent literature related to diabetes, therapeutic education and self-care.

\section{Methodology:}

A literature search was conducted PudMed, care, Ibecs, Lilacs, Scielo and Enfispo databases in February 2012. The time limit was 5 years and searched keywords in title and abstract as well as how descriptors.

\section{Findings:}

A total of 194 articles were identified. The 29 were included in the study $(n=54)$. The 64.4 works were located in PudMed $(n=124)$; not found any result in the database take care. The main reason for exclusion was that the articles were not directly related with the subject of the review in the cases $(n=65)$ 32.8. Work with qualitative character were the most abundant $(n=12)$.

\section{Conclusions:}

The implementation of innovative educational interventions focused on self-care seems to have benefits on diabetic patients, improving the management of the disease or the levels of glucose and glycosylated hemoglobin, facilitating the empowerment of patients or weaker parental control in diabetics type I. Further experimental research to clarify what kind of educational interventions have greater advantages and benefits for people with diabetes in different contexts is needed.

\section{Key words:}

diabetes mellitus, diabetes type I, diabetes type II, education, self-care. 


\section{INTRODUCCIÓN}

La Educación Terapéutica según la Organización Mundial de la Salud (OMS), comprende "el conjunto de actividades educacionales esenciales para la gestión de las enfermedades crónicas, llevadas a cabo por los profesionales de la salud formados en el campo de la educación, creadas para ayudar al paciente o grupos de pacientes y familiares a realizar su tratamiento y prevenir las complicaciones evitables, mientras se mantiene o mejora la calidad de vida" $(\text { OMS 1998) })^{1}$.

Ajustando esta definición, podemos encontrar que la Educación Terapéutica aplicada a una enfermedad crónica concreta como la Diabetes se debe tratar como un proceso con el que se facilita al paciente y a su familia el aprendizaje de las habilidades y conocimientos necesarios para asumir una actitud adecuada frente a su enfermedad, potenciando los autocuidados, así como el apoyo necesario para que puedan formar parte activa del tratamiento ${ }^{2}$.

Por otro lado, debemos tener en cuenta que el tratamiento de las enfermedades se basa en dos tipos de recursos: los fármacos y las normas de conducta. La Educación Terapéutica en Diabetes (ETD), influye en la variabilidad pronóstica de la diabetes, resaltando el hecho de que el rigor del profesional de la salud debería ser el mismo prescribiendo fármacos que dando normas de conducta ${ }^{3}$. Sólo visto de forma integrada, se apreciará el proceso que se remarcaba en el párrafo anterior.

Así, los profesionales de la salud responsables de la ETD deben tener una formación adecuada relacionada con: la enfermedad, la pedagogía, la psicología, habilidades de comunicación y competencia cultural, para ser capaces de transmitir la ETD tras adaptar las técnicas de enseñanza a los ritmos individuales de cada paciente ${ }^{2,3}$.

El objetivo del presente estudio consiste en revisar los estudios relacionados con la diabetes, la educación terapéutica y los autocuidados que 
aparecen en la literatura reciente. Como objetivos secundarios se han propuesto estudiar cuáles son las repercusiones que tiene la ETD sobre los pacientes y sobre sus autocuidados, así como la detección de posibles áreas de mejora en la investigación.

\section{MATERIAL Y MÉTODO}

Se realizó una búsqueda previa de documentos en Google Schoolar y posteriormente se concretaron los descriptores a utilizar para realizar la búsqueda bibliográfica sistematizada a través de una consulta con la aplicación DeCS (descriptores en ciencias de la salud) de la Biblioteca Virtual en Salud y de los Medical Subjects Headings (Mesh) de la Biblioteca Nacional de Estados Unidos, tras consenso por el grupo de investigación.

Se determinó la estrategia de búsqueda y se realizó una búsqueda bibliográfica en las bases de datos PudMed, Cuiden, Ibecs, Lilacs, Scielo y Enfispo en febrero de 2012. El límite temporal fue de 5 años y se buscaron las palabras clave en título y abstract, además de como descriptores. Se estableció un sistema de alertas en PubMed que avisara de nuevos resultados durante el desarrollo de la investigación. El resumen de la estrategia de búsqueda utilizada puede consultarse en la ilustración 1.

La revisión bibliográfica se realizó por pares en todas las bases de datos y se compararon los resultados obtenidos; los mismos investigadores intentaron recuperar el texto completo, o al menos el abstract, de cada uno de los artículos en diferentes fondos documentales. Otros dos investigadores seleccionaron por consenso los artículos que fueron incluidos en el estudio. Fueron excluidos aquellos que versaban sobre varias patologías, aquellos con formato de "paper" o ensayo, los que no se encontraban relacionados directamente con el objetivo del trabajo, los que no pudieron ser recuperados y los artículos anteriores a 2008. En caso de desacuerdo entre los investigadores encargados de la selección de los trabajos, la aceptación o rechazo de los artículos se realizó por consenso del grupo de investigación. 
Una vez seleccionados los artículos, éstos fueron separados en función de los descriptores utilizados en la búsqueda para diabetes mellitus y dependiendo de su naturaleza, se clasificaron en estudios cualitativos, descriptivos, cuasi experimentales, experimentales, revisiones sistemáticas, meta-análisis y otros $(N=1$, revisión bilbiográfica, etc). Se realizó la lectura crítica de los artículos por pares de investigadores y se elaboraron las conclusiones de la revisión bibliográfica.

Como limitaciones metodológicas, cabe decir que no se realizó el truncamiento de los descriptores utilizados, ni se llevó a cabo la revisión de las referencias bibliográficas de los artículos incluidos en la revisión bibliográfica.

\section{RESULTADOS}

Se identificaron un total de 194 artículos tras realizar la búsqueda en las diferentes bases de datos, incluyendo tres artículos a través del sistema de alertas de PubMed. De éstos, tras aplicar los criterios de exclusión, fueron aceptados a revisión el 29\% $(n=54)$. El $64.4 \%$ de los trabajos fueron localizados en PudMed ( $n=124)$; no se encontró ningún resultado en la base de datos CUIDEN. El principal motivo de exclusión de los artículos fue que no estaban relacionados directamente con el tema de la revisión en el $32.8 \%$ de los casos $(n=65)$. En la tabla 1 puede verse la distribución de los artículos recuperados en cada base de datos y la cantidad de artículos excluidos por motivos de exclusión.

La investigación cualitativa fue la más representativa del conjunto de artículos incluidos, para los descriptores empleados en la búsqueda, con un total de 19 artículos (34.55\%), seguida de las publicaciones con carácter descriptivo $(n=11)$ y de aquellas clasificadas como otros $(n=11)$, en el $20 \%$ de los casos para cada tipo.

Con el descriptor Diabetes Mellitus (DM), se localizaron un total de 8 
artículos, siendo la investigación con carácter cualitativo $(n=3)$ o experimental $(n=3)$ las más representativas con un $37.5 \%$ cada una de ellas. De los once artículos localizados con el descriptor Diabetes Mellitus tipo 1 (DM1) el 36\% se clasificó como investigación cualitativa $(n=4)$, y otro $36 \%$ con carácter descriptivo $(n=4)$. Los resultados para el descriptor Diabetes Mellitus tipo 2 (DM2) fueron los más representativos del conjunto de descriptores con un total de $36(65.45 \%)$ artículos incluidos en la revisión, tras aplicar los criterios de exclusión. Los trabajos con carácter cualitativo fueron los más abundantes $(n=12)$. No se localizó ningún artículo con los descriptores "Diabetes gestacional" ni "Estado pre-diabético". En la tabla 2 puede verse la relación entre el tipo de investigación y los descriptores utilizados para la búsqueda.

\section{DISCUSIÓN}

Obviando los resultados con los descriptores "Diabetes gestacional" y "Estado pre-diabético", los resultados del descriptor DM han sido los más escasos. Se han identificado dos estudios cualitativos; uno de ellos sobre las prácticas en auto-cuidados en pacientes diabéticos ${ }^{4}$ que evidencia la existencia de malas prácticas de autocuidados, sobretodo en los pacientes con peor control glicémico y otro trabajo que describe una intervención educativa en la que se elaboraron materiales educativos de forma conjunta con diabéticos, teniendo en cuenta sus experiencias en auto-cuidados ${ }^{5}$. Un tercer estudio realizó un análisis de regresión múltiple ${ }^{6}$ sobre diferentes factores que podían influir en la calidad de los autocuidados de los pacientes; el resultado de éste fue que el nivel educativo o de alfabetización (literacy) era el factor más relevante en este sentido, por lo que los autores recomendaban que se tuviera muy en cuenta a la hora de crear e implantar intervenciones educativas.

Uno de los tres ensayos clínicos identificados con el descriptor $\mathrm{DM}^{7}$ comparaba una intervención educativa tradicional con otra centrada en el automanejo de la enfermedad. Otro comparaba una intervención formativa on-line 
centrada en el manejo de la enfermedad ${ }^{8}$. El tercero comparaba los resultados de una intervención telefónica con la educación tradicional en base a la modificación de los auto-cuidados ${ }^{9}$. Los tres estudios presentaron mejoras en diferentes indicadores como nivel de glucosa basal, hemoglobina glicosilada, cantidad de ejercicio o adherencia al tratamiento, entre otros, en los grupos experimentales expuestos a las intervenciones educativas innovadoras centradas en los autocuidados.

Para el descriptor DM1 se han localizado pocos trabajos que comparen una intervención educativa, individual o grupal, centrada en los autocuidados con la educación tradicional. No se ha encontrado ningún estudio que compare los resultados de dos intervenciones educativas centradas en los autocuidados, ni ninguno que compare los resultados de una intervención de ETD individual con otra de ETD grupal. La mayoría de los trabajos encontrados son estudios descriptivos $^{10-13}$ y/o cualitativos ${ }^{14-17}$, a excepción de un estudio experimental ${ }^{18}$ y otro cuasi-experimental ${ }^{19}$ del tipo antes-después. También se ha recuperado una revisión bibliográfica ${ }^{20}$ que integra metodología cualitativa y cuantitativa.

A pesar de la poca evidencia identificada, los resultados de los trabajos parecen indicar que las intervenciones educativas innovadoras centradas en los autocuidados, producen mejores resultados tanto en el control de los niveles de glucemia, como de hemoglobina glicosilada, en pacientes diabéticos tipo I, mejorando el auto-manejo de la enfermedad y disminuyendo la necesidad de control paterno ${ }^{18,19}$, siempre en comparación con la educación tradicional.

Los estudios cualitativos sobre DM1, incluidos en esta revisión, versan sobre las experiencias de vida de niños y adolescentes con esta patología, así como sobre los diferentes obstáculos y factores que influyen en el empoderamiento y auto-manejo de la patología ${ }^{14-17}$. 
Los resultados aportados por la búsqueda del descriptor DM2 no han sido tan poco fructíferos. Se han encontrado cantidad de estudios relevantes que tratan de conocer cómo influyen las intervenciones de enfermería en el autocuidado de las personas con DM2 con apoyo educativo, explorando las influencias socio-culturales y el contexto social en la DM2 (desde los factores psicosociales hasta la edad, la familia y los amigos). Se ha podido vislumbrar cómo influye la visita domiciliaria en atención primaria para el autocuidado de la DM2 sobretodo en los ítems alimentación y actividad física; y se ha podido patentar que las intervenciones de enfermería mejoran los niveles de glucemia, de hemoglobina glicosilada, índice de masa corporal, los conocimientos y el peso, siendo éstas más influyentes que las intervenciones médicas por diversos motivos $^{21-35}$.

Aún así, se sigue echando en falta una comparación estructurada de ETD en autocuidados con empowerment frente a la educación tradicional, para la DM2. Es cierto que en este descriptor se han hallado resultados un poco más interesantes respecto a la educación, siendo éstos que la educación grupal estructurada, centrada en los autocuidados, debe utilizarse como complemento a la educación individual o que una intervención grupal es más efectiva con apoyo telefónico individual post-intervención; pero se continúa sin verificar si es más efectivo realizar una intervención grupal frente a una individual ${ }^{38-45}$.

Según parece, una intervención personalizada e individual puede ser más efectiva puesto que varios estudios avalan que en la DM2 se deben tener en cuenta para el auto-control de la misma: las influencias socio-culturales, el contexto social, los factores psico-sociales, la edad, la cultura, las creencias y las actitudes que se tienen frente al control de esta enfermedad y prevención de las posibles complicaciones potenciales, aunque no se ha hallado ningún estudio que lo avale de forma fehaciente ${ }^{40-56}$.

La cantidad y variedad de tipos de estudio encontrados con el descriptor 
DM2 es más extensa que en la DM1, permitiendo un nivel ligeramente superior de evidencia. Por otra parte, el hecho de no encontrar artículos con el descriptor "estado pre-diabético" obliga a reflexionar sobre la intervención de los profesionales de enfermería en la promoción de la salud. Así, se apoya que los profesionales de enfermería no tienen que esperar a que aparezca en la consulta otra persona con este problema de salud para aprender cómo funciona el glucómetro, sino que se puede emprender la educación terapéutica desde las personas con riesgo potencial de desarrollar esta enfermedad, promoviendo estilos de vida saludables, para reducir el impacto e incluso retrasar la aparición de ésta 57.

El presente estudio no está exento de limitaciones. Por una parte, la búsqueda se podría haber ampliado a otras bases de datos como Chochrane, CINHAL o ERIC. Por otra parte, no se ha realizado truncamiento en la búsqueda ni se han revisado las referencias bibliográficas de los artículos recuperados, lo que puede haber limitado la obtención y recuperación de artículos relevantes.

\section{CONCLUSIONES}

Las bases de datos generales son las que mayor cantidad de artículos recogen sobre este tema. La diabetes y la ETD, tanto en su vertiente asistencial como preventiva, es ampliamente tratada por la enfermería; en base a esto, cabe decir que llama la atención que las bases de datos específicas de enfermería sean las que contienen menor cantidad de resultados. La mayoría de los estudios recuperados son de tipo cualitativo.

Como se ha visto en esta revisión, la ETD centrada en los autocuidados parece ofrecer mejores resultados que la educación tradicional, por lo que se hace necesario llevar a cabo más estudios experimentales y revisiones sistemáticas con meta-análisis, que permitan clarificar que tipo de intervenciones educativas, centradas en los autocuidados, ofrecen más 
beneficios y ventajas a las personas con diabetes en diferentes contextos.

\section{BIBLIOGRAFÍA}

1. Rico, MA; Calvo, I; Díaz Gómez, J; Gimena, M. Educación terapéutica (ET) en pacientes con obesidad. Rev Esp Nutr Comunitaria 2008; 14 (3): 172-78.

2.Yoldi, C; Gómez, A. Educación terapéutica para pacientes en tratamiento con infusión subcutánea continua de insulina. Av Diabetol 2009; 25: 50712.

3. Figuerola, D. Manual de educación terapéutica en diabetes. Díaz de Santos: 2011.

4. Tan, MY; Margarey, J. Self.care practices of Malaysian adults wtih diabetes and sub-optimal glycaemic control. Patient Educ Couns. $2008 ; 72(2): 252-67$.

5. Wallace, AS; Carlson, JR; Malone, RM; Joyner, J; Dewalt, DA. The influence of literacy on patient-reported experiences of diabetes selfmanagement support. Nurs Res.2010;59(5):356-63.

6. Carvalho Torres, H; Abrantes Candido, N; Rodrigues Alexandre, L; Lobato Pereira, F. O proceso de elaboraçao do autocuidado no programa educativo em diabetes. Rev Bras Enferm. 2009(62(2):312-16.

7. Tan, MY; Margarey, JM; Chee, SS; Lee, LF; Tan MH. A brief structured programme enhances self-care practices and improves glycaemis control in Malasyan with pooly controlled diabetes. Health Education Research. 2011;26(5):896-907.

8. Carter, EL; Numlee-Bland, G; Callender, C. A patient-centric, providerassisted diabetes telehealth self-management intervention for urban minorities. Perspect Health Inf Manag. 2011;1(8):1b.

9. Amoako, E; Skelly, AH; Rossen, EK. Outcomes of an ntervention to reduce uncertainty among African women with diabetes. West J Nurs Res. 
2008;30(8):928-42.

10. Distiller, LA; Broown, MA; Joffe, I; Kramer, BD. Striving the impossible dream: a comunity-based multi-practice collaborative model of diabetes management. Diabet Med. 2010; 27(2): 197-202.

11. Keough. L; Sullivan-Bolyai, S; Crawford, S; Schilling, L; Dixon, J. Selfmanagement of type 1 diabetes across adolescence. Diabetes educ. 2011;37(4):486-500.

12. Chien, SC; Larson, E; Nakamura, N; Lin, SJ. Self-care problems of adolescents with type 1 diabetes in southern Taiwan. J Pediatr Nurs. 2007; 22(5):404-09.

13. Vázquez-Campo, $M$; Bande-Rodriguez, $M$; Pérez-Dinamarca, AP; Mouriño López, Y. Coping and Management of the disease in patients with diabetes mellitas type 1 . Enf Clín. 2011; 21(4): 210-13.

14. Wang, YL; Brown, SA; Horner, SD. School-based lived experiences of adolescents with type 1 diabetes: a preliminary study. J Nurs Res. 2010;18(4):258-65.

15. Rasmussen, B; Ward, G; Jenkins, A; King, SJ; Dunning, T. Young adult's management of type 1 diabetes during life transitions. J Clin Nurs. 2011;20(13-14):1981-92.

16. Jonsson, L; Hallstrom, I; Lundqvist, A. A multi-disciplinary education process related to the discharging of children from hospital when the cild has been diagnosed with type 1 diabetes. A qualitative study. BMC Pediatrics. 2010; 10:36.

17. Dympna, C; Murphy, K; Laxton, J; White, FF; Dineen, S. A longitudinal qualitative study examining the factors impacting on the ability of persons with T1DM to assimilate the Dose Adjustment for Normal Eating (DAFNE) principles into daily living and how these factors change over time. BMC Public Health. 2011; 11:672.

18. Grey, $M$ et al. Effects of coping skills training in school-age children with type 1 diabetes. Res Nurs Health. 2009;32(4): 405-18.

19. Hernandez, CA; Hume, MR; Rodger, W. Evaluation of a self-awareness intervention for type 1 diabetes and hipoglicemia unawareness. CJNR. 2008;40(3): 38-56. 
20. Kelo, M; Martikaimen, M; Eriksson, E. Self-care of school-age children with diabetes: an integrative review. 2011;67(10):2096-108.

21. Avila Alpirez, H; Meza Guevara, S; Frías Reyna, B; Sánchez Andrade, E; Vega Alanís, C; Hernández Saldivar, MA. Intervención de enfermería en el autocuidado con apoyo educativo en personas con diabetes mellitus tipo 2. Cultura de los cuidados 2006; 20: 141-46.

22. Wiler, D; Crist, JD. Diabetes Self-Management in a Latino Social Environment. The Diabetes Educator. 2009; 35(2):285-92.

23. Torres, HC; Roque, C; Nunes, C. Home visits: an educational strategy for self careo $f$ diabetic clients in primary care. Rev. Enferm. UERJ 2011;19(1): 89-93.

24. Vincent D, Pasvogel A, Barrera L. A feasibility study of a culturally tailored diabetes intervention for Mexican Americans. Biol Res Nurs. 2007;9(2):130-41.

25. Welch G, Allen NA, Zagarins SE, Stamp KD, Bursell SE, Kedziora RJ. Comprehensive diabetes management program for poorly controlled Hispanic type 2 patients at a community health center. Diabetes Educ. 2011 Sep-Oct; 37(5):680-8.

26. Sigurdardottir $A K$, Benediktsson $\mathrm{R}$, Jonsdottir $\mathrm{H}$. Instruments to tailor care of people with type 2 diabetes. J Adv Nurs. 2009;65(10):2118-30.

27. Sixta CS, Ostwald S. Texas-Mexico border intervention by promotores for patients with type 2 diabetes. Diabetes Educ. 2008;34(2):299-309.

28. Hyun KS, Kim KM, Jang SH. The effects of tailored diabetes education on blood glucose control andself-care. J Korean Acad Nurs. 2009t;39(5):720-30

29. Polzer RL. African Americans and diabetes: spiritual role of the health care provider in self-management. Res Nurs Health. 2007;30(2):164-74.

30. Gatt S, Sammut R. An exploratory study of predictors of self-care behaviour in persons with type 2 diabetes. Int J Nurs Stud. 2008;45(10):1525-33.

31. Song $M$, Lee $M$, Shim B. Barriers to and facilitators of selfmanagement adherence in Korean older adults with type 2 diabetes. Int J Older People Nurs. 2010;5(3):211-8. 
32. Johnson-Spruill I, Hammond P, Davis B, McGee Z, Louden D. Health of Gullah families in South Carolina with type 2 diabetes: diabetes selfmanagement analysis from project SuGar. Diabetes Educ. 2009;35(1):117-23.

33. George SR, Thomas SP. Lived experience of diabetes among older, rural people. J Adv Nurs. $2010 ; 66(5): 1092-100$.

34. Lippa KD, Klein HA. Portraits of patient cognition: how patients understand diabetes self-care. Can J Nurs Res. 2008;40(3):80-95.

35. Bundesmann R, Kaplowitz SA. Provider communication and patient participation in diabetes self-care. Patient Educ Couns. 2011;85(2):143-7. 36. Wu SF, Huang YC, Liang SY, Wang TJ, Lee MC, Tung HH. Relationships among depression, anxiety, self-care behaviour and diabetes education difficulties in patients with type- 2 diabetes: a cross-sectional questionnaire survey. Int J Nurs Stud. 2011;48(11):1376-83.

37. Jordan DN, Jordan JL. Self-care behaviors of Filipino-American adults with type 2 diabetes mellitus. J Diabetes Complications. 2010;24(4):2508.

38. Bai $Y L$, Chiou $C P$, Chang YY. Self-care behaviour and related factors in older people with Type 2 diabetes. J Clin Nurs. 2009;18(23):3308-15.

39. Wu SF, Courtney M, Edwards H, McDowell J, Shortridge-Baggett LM, Chang PJ. Self-efficacy, outcome expectations and self-care behaviour in people with type 2. J Clin Nurs. 2007;16(11C):250-7.

40. Heinrich E, Candel MJ, Schaper NC, de Vries NK. Effect evaluation of a Motivational Interviewing based counselling strategy in diabetes care. Diabetes Res Clin Pract. 2010;90(3):270-8.

41. Moriyama M, Nakano M, Kuroe Y, Nin K, Niitani M, Nakaya T. Efficacy of a self-management education program for people with type 2 diabetes: results of a 12 month trial. Jpn J Nurs Sci. 2009;6(1):51-63.

42. Kumar CP. Application of Orem's self-care deficit theory and standardized nursing languages in a case study of a woman with diabetes. Int J Nurs Terminol Classif. 2007;18(3):103-10.

43. Jones RA, Utz SW, Williams IC, Hinton I, Alexander G, Moore C, Blankenship J, Steeves R, Oliver N. Family interactions among African 
Americans diagnosed with type 2 diabetes. Diabetes Educ. 2008;34(2):318-26.

44. González Marcos, M; Perpinyà, D; Melció, D; Casellas, P; Mir, S; García, MT. Efectividad de un programa de educación grupal estructurada en personas con diabetes mellitus tipo 2. Enfermería clínica 2005;15:141146.

45. Distiller LA, Brown MA, Joffe BI, Kramer BD. Striving for the impossible dream: a community-based multi-practice collaborative model of diabetes management. Diabet Med. 2010;27(2):197-202.

46. Xu Y, Pan W, Liu H. Self-management practices of Chinese Americans with type 2 diabetes. Nurs Health Sci. 2010;12(2):228-34.

47. Borges WJ, Ostwald SK. Improving foot self-care behaviors with Pies Sanos. West J Nurs Res. 2008;30(3):325-41; discussion 342-9.

48. Shi Q, Ostwald SK, Wang S. Improving glycaemic control self-efficacy and glycaemic control behaviour in Chinese patients with type 2 diabetes mellitus: randomised controlled trial. J Clin Nurs. 2010;19(3-4):398-404.

49. D'Eramo Melkus G, Chyun D, Vorderstrasse A, Newlin K, Jefferson V, Langerman $\mathrm{S}$. The effect of a diabetes education, coping skills training, and care intervention on physiological and psychosocial outcomes in black women with type 2 diabetes. Biol Res Nurs. 2010;12(1):7-19.

50. Vincent D. Culturally tailored education to promote lifestyle change in Mexican Americans with type 2 diabetes. J Am Acad Nurse Pract. 2009;21(9):520-7.

51. Utz SW, Williams IC, Jones R, Hinton I, Alexander G, Yan G, Moore C, Blankenship J, Steeves R, Oliver MN. Culturally tailored intervention for rural African Americans with type 2 diabetes. Diabetes Educ. 2008;34(5):854-65.

52. Leyva B, Zagarins SE, Allen NA, Welch $G$. The relative impact of diabetes distress vs depression on glycemic control in hispanic patients following a diabetes self-management education intervention. Ethn Dis. 2011;21(3):322-7.

53. McEwen MM, Pasvogel A, Gallegos G, Barrera L. Type 2 diabetes selfmanagement social support intervention at the U.S.-Mexico border. Public 
Health Nurs. 2010;27(4):310-9.

54. Hayes E, McCahon C, Panahi MR, Hamre T, Pohlman K. Alliance not compliance: coaching strategies to improve type 2 diabetes outcomes. J Am Acad Nurse Pract. 2008;20(3):155-62.

55. Nam S, Chesla C, Stotts NA, Kroon L, Janson SL. Barriers to diabetes management: patient and provider factors. Diabetes Res Clin Pract. 2011;93(1):1-9.

56. He X, Wharrad HJ. Diabetes knowledge and glycemic control among Chinese people with type 2 diabetes. Int Nurs Rev. 2007;54(3):280-7. 57. Mena Tudela, D. La diabetis, una malaltia que es pot prevenir? Cap i Cua 2011;19:5. 
Tabla 1: Resultados búsqueda.

\begin{tabular}{|c|c|c|c|c|c|c|c|}
\hline \multirow[b]{2}{*}{$\begin{array}{c}\text { Base de } \\
\text { datos }\end{array}$} & \multicolumn{2}{|c|}{ Artículos } & \multicolumn{5}{|c|}{ Artículos excluidos por criterios de exclusión } \\
\hline & Búsqueda & Incluidos & Paper & $\begin{array}{c}\text { Otras } \\
\text { patologías }\end{array}$ & $\begin{array}{c}\text { Año } \\
\text { publicación }\end{array}$ & $\begin{array}{c}\text { No } \\
\text { recuperados }\end{array}$ & $\begin{array}{c}\text { No tema } \\
\text { estudio }\end{array}$ \\
\hline PubMed & $124(64.4 \%)$ & $46(85.5 \%)$ & $23(96 \%)$ & $9(81.8 \%)$ & 0 & 0 & $46(70.9 \%)$ \\
\hline Lilacs & $58(29.29 \%)$ & $3(5.5 \%)$ & $1(4 \%)$ & $2(18.2 \%)$ & $34(90 \%)$ & 0 & $18(27.6 \%)$ \\
\hline Cuiden & 0 & 0 & 0 & 0 & 0 & 0 & 0 \\
\hline Ibecs & $7(3.53 \%)$ & $1(1.8 \%)$ & 0 & 0 & $6(10 \%)$ & 0 & 0 \\
\hline Enfispo & $1(0.5 \%)$ & $1(1.8 \%)$ & 0 & 0 & 0 & 0 & 0 \\
\hline Scielo & $1(0.5 \%)$ & $1(1.8 \%)$ & 0 & 0 & 0 & 0 & 0 \\
\hline Alertas & & & & & & & \\
\hline PubMed & $3(1.5 \%)$ & $2(3.64 \%)$ & 0 & 0 & 0 & 0 & $1(1.5 \%)$ \\
\hline TOTALES & $194(100 \%)$ & $54(29 \%)$ & $24(12.1 \%)$ & $11(5.5 \%)$ & $40(20.2 \%)$ & 0 & $65(32.8 \%)$ \\
\hline
\end{tabular}

Tabla 2: Tipo de investigación según descriptores de diabetes.

\begin{tabular}{|c|c|c|c|c|c|c|c|c|}
\hline \multicolumn{9}{|c|}{ Descriptores de diabetes mellitus } \\
\hline \multirow[t]{2}{*}{ Tipo de investigación } & \multicolumn{2}{|c|}{ Diabetes mellitus (DM) } & \multicolumn{2}{|c|}{ DM tipo 1} & \multicolumn{2}{|c|}{ DM tipo 2} & \multicolumn{2}{|c|}{ TOTALES } \\
\hline & n & $\%$ & $\mathbf{n}$ & $\%$ & n & $\%$ & n & $\%$ \\
\hline Cualitativa. & 3 & $37.5 \%$ & 4 & $36 \%$ & 12 & $33,33 \%$ & 19 & $34.55 \%$ \\
\hline Descriptiva & 0 & $0 \%$ & 4 & $36 \%$ & 7 & $19,44 \%$ & 11 & $20 \%$ \\
\hline Experimental & 3 & $37.5 \%$ & 1 & $9 \%$ & 4 & $11,11 \%$ & 8 & $14.55 \%$ \\
\hline Cuasi-experimental & 0 & $0 \%$ & 1 & $9 \%$ & 2 & $5,55 \%$ & 3 & $5.45 \%$ \\
\hline Revisión sistemática & 0 & $0 \%$ & 0 & $0 \%$ & 2 & $5,55 \%$ & 2 & $3.63 \%$ \\
\hline Meta-análisis & 0 & $0 \%$ & 0 & $0 \%$ & 0 & $0 \%$ & 0 & $0 \%$ \\
\hline Otros & 1 & $12.5 \%$ & 1 & $9 \%$ & 9 & $25 \%$ & 11 & $20 \%$ \\
\hline TOTALES & 7 & $14.55 \%$ & 11 & $20 \%$ & 36 & $65.45 \%$ & 54 & $100 \%$ \\
\hline
\end{tabular}


Ilustración 1. Estrategia de búsqueda y metodología

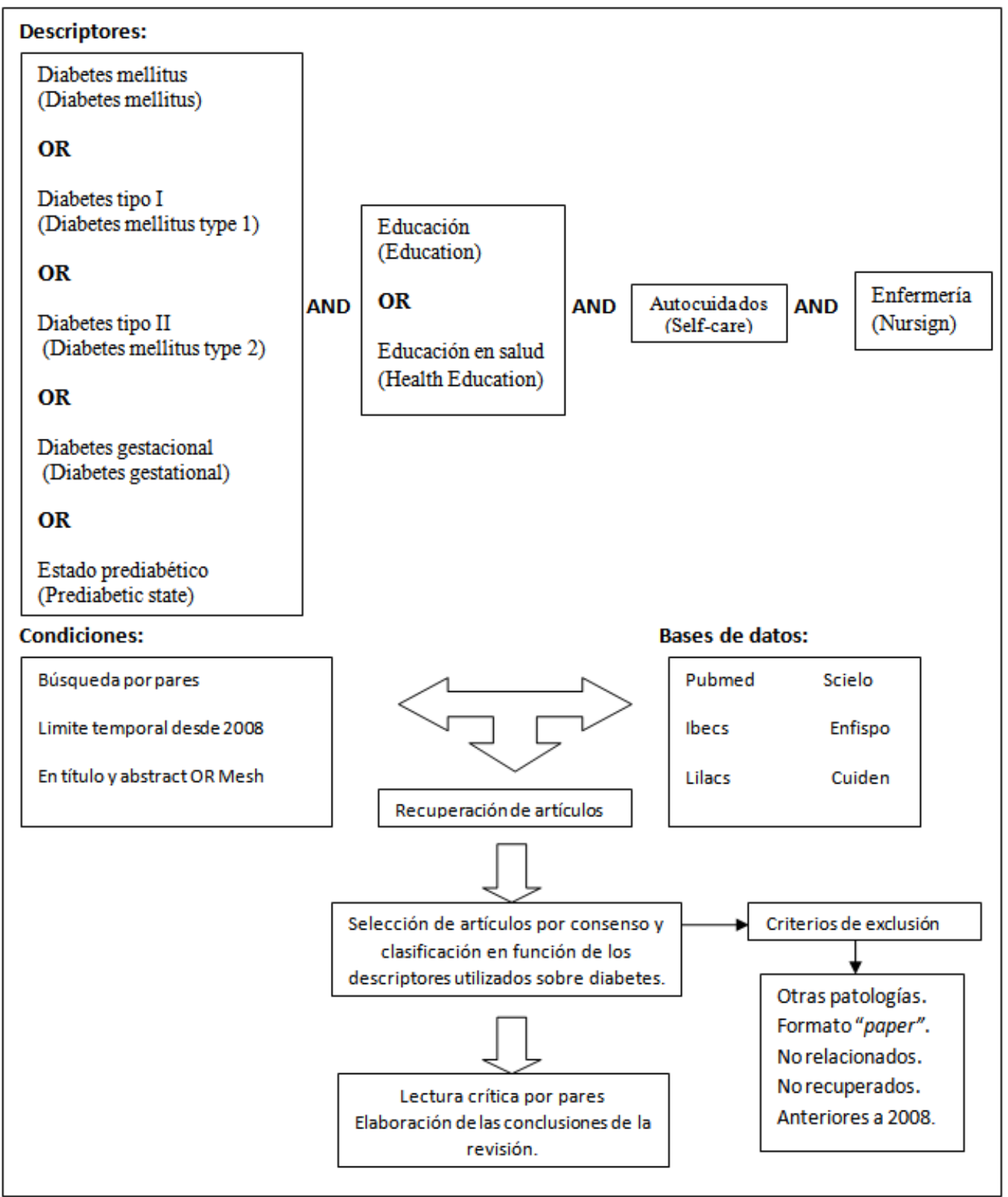

